Enfermagem Brasil 2018;17(5):471-9

\title{
ARTIGO ORIGINAL \\ Telessaúde como suporte na assistência da enfermagem em obstetrícia
}

Danielle Santos Alves, M.Sc.*, Natália Maria Penha Coutinho, M.Sc. ${ }^{* *}$, Priscila Santos Alves, M.Sc. ${ }^{* \star}$, Erika Maria Alves da Silva ${ }^{\star \star * \star}$, Magdala de Araújo Novaes, D.Sc. ${ }^{* \star \star \star}$

${ }^{*}$ Enfermeira Obstétrica, Professora Adjunta do Departamento de Enfermagem (UFPE), Doutoranda do Programa de Pós-Graduação do Centro de Informática da UFPE; ${ }^{* \star}$ Enfermeira especialista em Saúde da Família e em Análise de situação de saúde, Teleconsultora do Núcleo de Telessaúde- Hospital das Clínicas/UFPE e inspetora sanitária do Município de Olinda/PE, ${ }^{* *}$ Enfermeira Obstétrica, Professora Substituta do Departamento de Enfermagem da UFPE, ${ }^{* * *}$ Enfermeira, Aluna de Iniciação Científica do Núcleo de Telessaúde de Pernambuco NUTES/UFPE, ${ }^{* * * * *}$ Professora associada de Informática em Saúde do Departamento de Medicina Clínica e docente do Curso de Medicina da UFPE. Fundadora e coordenadora do Grupo de Pesquisa de Tecnologias da Informação em Saúde (TIS) e do Núcleo de Telessaúde (NUTES) da UFPE

Recebido em 22 de novembro de 2017; aceito em 16 de maio de 2018.

Endereço de correspondência: Danielle Santos Alves, UFPE, Av. Prof. Moraes Rego, 844900, Cidade Universitária, Recife PE, E-mail: angeldannyalves@gmail.com; Natália Maria Penha Coutinho: natalia.coutinho@nutes.ufpe.br; Priscila Santos Alves: priscilastalves@gmail.com; Erika Maria Alves da Silva: contatoerikaalves@outlook.com; Magdala de Araújo Novaes: magdala.novaes@nutes.ufpe.br.

\section{Resumo}

Introdução: A teleassistência mostra-se capaz de melhorar a tomada de decisões compensando a escassez de recursos de cuidados de saúde em áreas específicas. Objetivo: Descrever a experiência da teleassistência na área de enfermagem em obstetrícia realizada pelo NUTES/UFPE. Métodos: Estudo descritivo, realizado com teleconsultorias de obstetrícia do NUTES/UFPE de 2009 a 2015. A partir de uma base de dados que reúne todas as teleconsultorias realizadas pelo NUTES, foram selecionadas todas as teleconsultorias cuja especialidade foi a obstetrícia. Resultados: Foram realizadas 906 teleconsultorias na área de obstetrícia. Os profissionais que mais utilizam e mais respondem a teleassistência são os enfermeiros com $55 \%$ e $66,9 \%$, respectivamente. Observa-se que $77 \%$ eram relacionadas com aspectos da gravidez e 17\% ao período de pós-parto. Conclusão: A teleassistência apoia os profissionais que necessitam de uma segunda opinião dos especialistas. Tem potencial de redução da morbimortalidade materna e neonatal. A enfermagem obstétrica pode apoiar os profissionais não especialistas a melhor atender às mulheres em todo o ciclo gravídico puerperal, desde à pré-concepção até o término do puerpério, estendendo-se também à atenção aos fetos e familiares.

Palavras-chave: telemedicina, enfermagem obstétrica, atenção primária à saúde, consulta remota.

\footnotetext{
Abstract

Telehealth as support in nursing care in obstetrics

Introduction: Teleassistance is capable to improve decision-making by compensating for the scarcity of health care resources in specific areas. Objective: To describe the experience of teleassistance in obstetrics nursing carried out by NUTES/UFPE. Methods: Descriptive study related to the NUTES/UFPE obstetrics teleconsultories from 2009 to 2015. From a database that gathers all the teleconsultories, we selected all teleconsultations whose specialty was obstetrics. Results: 906 teleconsultations were performed in the obstetrics area. The professionals who use the most and most respond to teleassistance are the Nurses with $55 \%$ and $66.9 \%$, respectively. It is observed that $77 \%$ was related to aspects of pregnancy and $17 \%$ to the postpartum period. Conclusion: Teleassistance supports professionals who need a second opinion from specialists. It has the potential to reduce maternal and neonatal morbidity and mortality. Obstetric nursing can support non-specialist professionals to better serve women
} 
throughout the puerperal pregnancy cycle, from preconception to the end of the puerperium, also extending attention to the fetus and family.

Key-words: telemedicine, obstetric nursing, primary health care, remote consultation.

\section{Resumen \\ Telemedicina como soporte en la asistencia de la enfermería en obstetricia}

Introducción: La teleasistencia se muestra capaz de mejorar la toma de decisiones compensando la escasez de recursos de atención de salud en áreas específicas. Objetivo: Describir la experiencia de la teleasistencia en el área de enfermería en obstetricia realizada por el NUTES/UFPE. Método: Estudio descriptivo, realizado con teleconsultorias de obstetricia del NUTES/UFPE de 2009 a 2015. A partir de una base de datos que reúne todas las teleconsultorias realizadas por el NUTES, fueron seleccionadas todas las teleconsultorias cuya especialidad fue la obstetricia. Resultados: Se realizaron 906 teleconsultorias en el área de obstetricia. Los profesionales que más utilizan y más responden la teleasistencia son los enfermeros con $55 \%$ y $66,9 \%$, respectivamente. Se observa que el $77 \%$ estaba relacionado con aspectos del embarazo y el $17 \%$ al período de posparto. Conclusión: La teleasistencia apoya a los profesionales que necesitan una segunda opinión de los expertos. Tiene potencial de reducción de la morbimortalidad materna y neonatal. La enfermería obstétrica puede apoyar a los profesionales no especialistas a mejor atender a las mujeres en todo el ciclo gravídico puerperal, desde la pre-concepción hasta el término del puerperio, extendiéndose también a la atención a los fetos y familiares.

Palabras-clave: telemedicina, enfermería obstétrica, atención primaria a la salud, teleconsultoria.

Introdução

A saúde da mulher é uma das prioridades de assistência à saúde no Brasil e no mundo. No âmbito mundial, esse tema ganha força e espaço e está presente em diversos acordos internacionais que possuem como objetivo a melhoria dos índices de saúde desse público. Apesar dos avanços dos últimos 25 anos em relação às políticas públicas relacionadas à saúde da mulher, ainda faz necessários avanços, especialmente nas ações referentes à redução da morbimortalidade de mulheres em idade fértil $[1,2]$.

A enfermagem é uma profissão que tem como foco principal a saúde e a qualidade de vida da pessoa, atuando na promoção, prevenção, recuperação e reabilitação da saúde. No cenário obstétrico, a participação do enfermeiro em políticas da atenção à saúde da mulher torna-se essencial, com papel fundamental na detecção precoce de doenças e agravos e, sobretudo, na educação em saúde visando à integralidade da assistência [1]. Para que o cuidado à mulher seja efetivo, é importante que este profissional esteja em constante atualização e tenha o suporte de outros níveis de assistência (secundária e terciária) [3].

A comunicação interinstitucional, entre os diferentes níveis de assistência à saúde, nem sempre é possível para os profissionais, tampouco para os usuários do serviço. A distância geográfica entre as unidades de nível primário e os centros de referência, dificulta a consulta a especialistas e, portanto, traz impactos negativos na condução da assistência. Outro obstáculo é o deslocamento dos usuários na busca por um cuidado especializado, que os expõe aos perigos durante o translado. Além disso, necessitam aguardar filas de espera para atendimento. Sendo assim, uma alternativa capaz de oferecer subsídios para diminuir a distância entre profissionais e pacientes dos níveis secundário e terciário de atenção à saúde é o uso da telessaúde [4].

A telessaúde consiste no uso de tecnologias da informação e comunicação (TIC) para facilitar a troca de informações clínicas e aprimoramento educacional entre os profissionais de saúde geograficamente separados [5,4]. Sua utilização permite que os profissionais tenham acesso a especialistas localizados remotamente (teleassistência) e proporciona interação entre estes para discussão de casos clínicos, questões de trabalho e/ou dúvidas clínicas (teleconsultorias) [6,7]. No Brasil, o telessaúde Brasil Redes incentiva a utilização desta tecnologia em diversos estados. Essa estratégia de saúde tem se mostrado eficaz e com resultados animadores [4].

Algumas experiências regionais demonstram a eficácia e a qualidade dos serviços de teleassistência $[7,8]$. Os resultados clínicos dos pacientes que fizeram uso dessa tecnologia corroboram e concluem que esses resultados são comparáveis ao acompanhamento tradicional de consultório presencial [9]. Sendo assim, a teleassistência mostra-se capaz de 
melhorar a tomada de decisões em situações agudas, compensando a escassez local de recursos de cuidados em áreas específicas e fornece acesso a especialistas [9].

Dentre as diversas áreas que podem ser beneficiadas pelo uso desta tecnologia, destaca-se a obstetrícia. É sabido que o ciclo gravídico-puerperal impõe sobre o organismo materno, diversas modificações. Estas, em sua maioria, transcorrem de maneira gradativa e fisiológica, de maneira a proporcionar o desenvolvimento saudável da mãe e do concepto. Através da teleassistência, os profissionais não especialistas podem solicitar apoio a obstetras na condução de casos onde ocorram intercorrências na gravidez de risco habitual, acompanhamento na gravidez de risco e auxílio no puerpério. Portanto, a teleassistência traz como principal benefício à integralidade do cuidado quando contribui na detecção precoce de doenças e agravos e na redução da morbimortalidade materna e neonatal [1].

Este estudo objetiva descrever a experiência na teleassistência na área de enfermagem em obstetrícia realizada pelo Núcleo de Telessaúde da Universidade Federal de Pernambuco (NUTES/UFPE).

Material e métodos

Estudo do tipo descritivo, quantitativo e transversal. A adoção do método quantitativodescritivo deve-se à sua possibilidade de realizar uma investigação com a finalidade de delinear, ou analisar, as características de fatos ou fenômenos, além de conhecer e controlar variáveis, preocupando-se com a representação numérica, com a medição objetiva e a quantificação de resultados, porém, com a possibilidade de discussão qualitativa destes [10].

$\mathrm{O}$ estudo foi realizado com base nas teleconsultorias da área de obstetrícia produzidas pelo Núcleo de Telessaúde da Universidade Federal de Pernambuco (NUTES/UFPE) entre os anos de 2009 e 2015. O NUTES/UFPE, localizado no Hospital das Clínicas da UFPE, atua nas áreas de telessaúde junto aos diversos municípios do estado de Pernambuco parceiros do Telessaúde Brasil. Esses profissionais solicitantes podem trocar experiências e requerer uma segunda opinião a especialistas teleconsultores (teleassistência) através dos seminários ou Plataforma de Telessaúde HealthNET [11,12].

As teleconsultorias são enviadas através da Plataforma HealthNet ou são feitas durante as discussões nos seminários da RedeNUTES. Estas são divididas em questões clínicas, casos clínicos e processo de trabalho. Nos seminários, as respostas são fornecidas imediatamente. Para as teleconsultorias enviadas pela Plataforma têm o prazo de até 48 horas úteis para ter o seu retorno efetuado.

A partir de uma base de dados que reúne todas as teleconsultorias realizadas pelo NUTES, foram selecionadas aquelas cuja especialidade foi a obstetrícia. Foram excluídas as que não se relacionavam com esta área. Os dados selecionados foram separados em uma planilha Microsoft Excel® onde foram realizadas as devidas análises estatísticas e geradas as tabelas e gráficos correspondentes aos resultados obtidos.

Foram analisadas as variáveis referentes a caracterização de ano, média de dias, canal de comunicação (Teleconsultoria individual: solicitante-teleconsultor; Coletiva: durante os seminários; ou através da Plataforma Healthnet), natureza e área da teleconsultoria, ocupação do solicitante e do teleconsultor, se houve resolução na primeira consultoria, percentual dos encaminhamentos, média de dias de retorno e grau de satisfação no uso do serviço. Também foram analisadas as categorias dentro da obstetrícia a qual as dúvidas se referiram e quais os respectivos descritores.

Foi traçado um perfil das teleconsultorias, medindo-se as frequências absolutas e relativas das variáveis do serviço, acompanhadas pelo Sistema de Monitoramento e Avaliação dos Resultados do Telessaúde/MS. Para caracterizar as teleconsultorias de acordo com a temática discutida, cada teleconsultoria foi recategorizada com descritores da Biblioteca Virtual de Saúde, após a leitura da dúvida do solicitante. Os descritores referem-se ao tema geral da dúvida (período pré-concepcional, gravidez, parto e período pós-parto), ao tema específico da dúvida em obstetrícia e à atividade da saúde apoiada pela teleconsultoria.

Este estudo está respaldado e obedece as normas da na portaria № 2.546 , de 27 de outubro de 2011 que regulamenta o Programa Telessaúde Brasil Redes [13], e foi aprovado pelo Comitê de Ética do Centro de Pesquisas Ageu Magalhães (CEP-CPqAM/Fiocruz) com registro no CAAE no 02791212.9.0000.5190, parecer nํ2 24/2012. 
Entre 2009 e 2015 foram solicitadas 10.868 teleconsultorias. Destas, 906 solicitações referiam-se a área de Obstetrícia, o que representou $8 \%$ do total. O ano de 2012 teve o maior número de solicitações em comparação com os outros anos $(30,8 \%)$. O tempo médio de resposta aos profissionais de origem ficou menor que um dia (24 horas).

Parte significativa das teleconsultorias foi realizada durante os seminários $(53,8 \%)$, quando as dúvidas eram esclarecidas coletivamente. Outra possibilidade de acesso foi a Plataforma Healthnet $(41,6 \%)$ ou a partir de webconferência individual $(4,6 \%)$. As teleconsultorias são categorizadas em: Questão Clínica (relacionadas às ações de saúde de um modo geral); Caso clínico (ligadas à assistência ao paciente); ou relativas aos Processos de trabalho das equipes de saúde da família. Em sua maioria, estas se relacionavam à questão clínica $(63,2 \%)$, seguidas por casos clínicos $(34,5 \%)$. A área de atendimento mais solicitada foi a Enfermagem (73,2\%), seguidas por Medicina (15,7\%). Algumas dúvidas enviadas eram multidisciplinares, envolvendo nutrição e fisioterapia $(2,1 \%)$ e enfermagem $(1,8 \%)$.

Observa-se que os profissionais que mais utilizam a teleassistência são os enfermeiros $(55 \%)$ e os agentes comunitários de saúde $(18,9 \%)$. A categoria profissional do teleconsultor que mais respondem a essas solicitações é o enfermeiro (66,9\%), seguido do médico (13,0\%). E importante enfatizar que muitas teleconsultorias $(7 \%)$ foram respondidas de modo multidisciplinar, englobando, em geral, a enfermagem e outras áreas do saber (Tabela I).

Tabela I - Caracterização das teleconsultorias realizadas pelo NUTES/UFPE. Recife/PE, 2017.

\begin{tabular}{|c|c|c|}
\hline Ano & $(\mathrm{n}=906)$ & $\%$ \\
\hline 2010 & 258 & 28,5 \\
\hline 2009 & 141 & 15,6 \\
\hline 2011 & 140 & 15,5 \\
\hline 2012 & 279 & 30,8 \\
\hline 2013 & 20 & 2,2 \\
\hline 2014 & 6 & 0,7 \\
\hline 2015 & 62 & 6,8 \\
\hline Dias de retorno da resposta & $(n=906)$ & \\
\hline Média de dias (dias) & 0,96 & \\
\hline Canal de comunicação & $(n=906)$ & $\%$ \\
\hline Teleconsultoria por Web. - Coletiva & 487 & 53,8 \\
\hline Healthnet & 377 & 41,6 \\
\hline Teleconsultoria por Web. - Individual & 42 & 4,6 \\
\hline Natureza da teleconsultoria & $(n=906)$ & $\%$ \\
\hline Questão Clínica & 573 & 63,2 \\
\hline Caso Clínico & 313 & 34,5 \\
\hline Processo de Trabalho & 20 & 2,2 \\
\hline Área de teleconsultoria & $(n=906)$ & $\%$ \\
\hline Enfermagem & 663 & 73,2 \\
\hline Medicina & 142 & 15,7 \\
\hline Nutrição & 21 & 2,3 \\
\hline Fisioterapia & 19 & 2,1 \\
\hline Fisioterapia e Nutrição & 19 & 2,1 \\
\hline Enfermagem e Nutrição & 16 & 1,8 \\
\hline Outras Áreas & 26 & 3,0 \\
\hline Ocupação do solicitante & $(n=906)$ & $\%$ \\
\hline Enfermeiro & 498 & 55,0 \\
\hline Agente comunitário de saúde & 171 & 18,9 \\
\hline Técnico em enfermagem & 58 & 6,4 \\
\hline Médico & 25 & 2,8 \\
\hline Outros profissionais de saúde & 36 & 3,9 \\
\hline Cirurgião dentista & 19 & 2,1 \\
\hline Não informado & 99 & 10,9 \\
\hline Ocupação do teleconsultor & $(n=906)$ & $\%$ \\
\hline Enfermeiro & 606 & 66,9 \\
\hline Médico & 118 & 13,0 \\
\hline Multidisciplinar & 63 & 6,9 \\
\hline Nutricionista & 21 & 2,3 \\
\hline Fisioterapeuta & 19 & 2,1 \\
\hline Cirurgião dentista & 11 & 1,2 \\
\hline Outros profissionais & 10 & 1,2 \\
\hline Não classificado & 58 & 6,4 \\
\hline Total geral & 906 & \\
\hline
\end{tabular}


Tabela II - Caracterização das teleconsultorias realizadas pelo NUTES/UFPE segundo o seu potencial de resolução e qualificação dos encaminhamentos. Recife/PE, 2017.

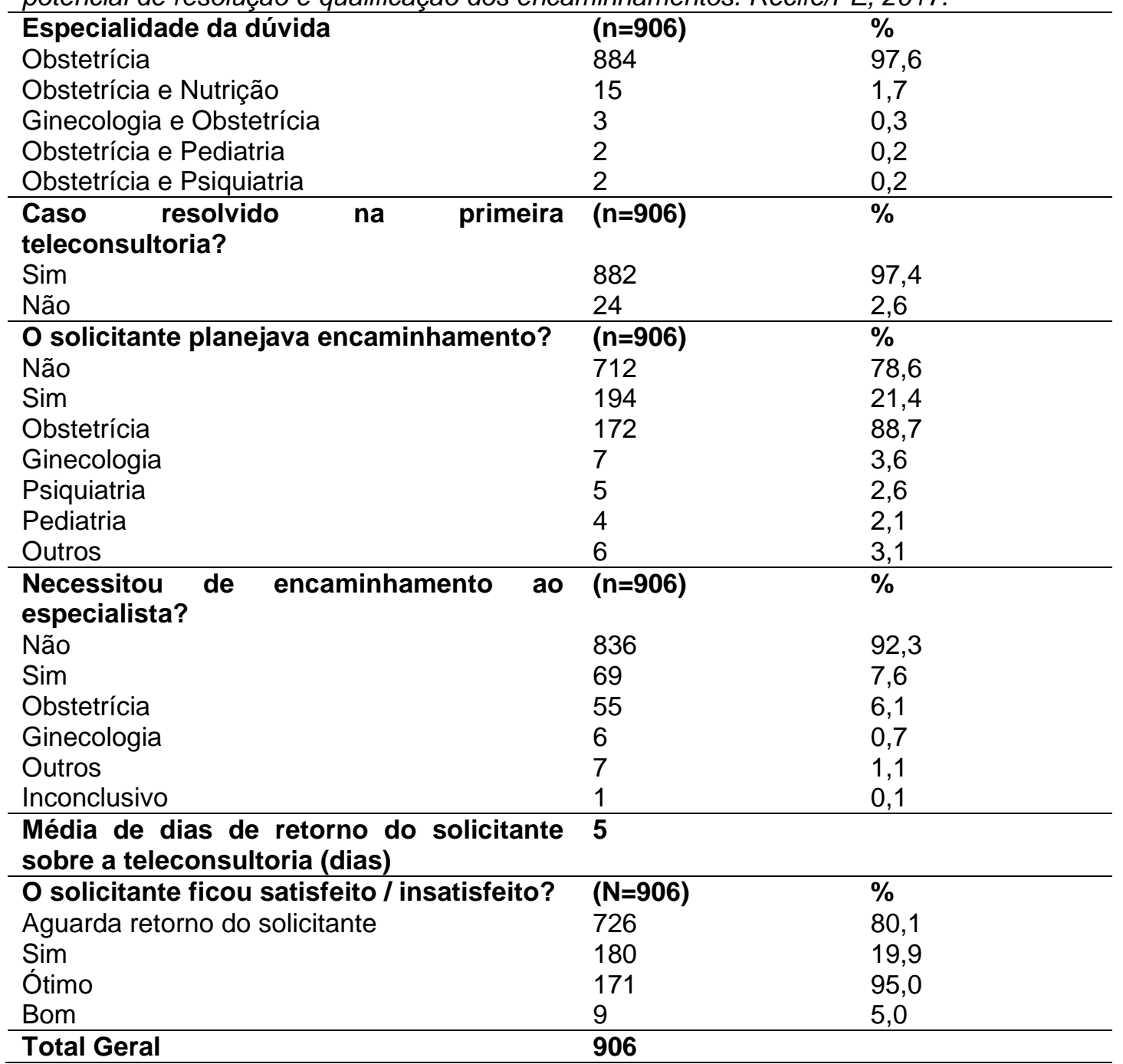

Com relação à temática geral da obstetrícia discutida, observa-se que $77,37 \%$ destas relacionavam-se com aspectos da gravidez e 16,78\% ao período de pós-parto. Apenas 4,42\% estavam relacionadas ao parto e 1,32\% à gravidez na adolescência. Menos de $1 \%$ não foram classificadas em nenhuma dessas áreas. A categorização das teleconsultorias foi organizada de acordo com os descritores DeCS/BVS (Tabela III).

Observa-se que na Categoria "Gravidez", o descritor mais usado foi o relacionado aos cuidados de Pré-Natal, seguidos de nutrição na gestação e hipertensão gestacional. Em "Gravidez na adolescência", a maior preocupação era relacionada às complicações inerentes a faixa etária. Na categoria "Parto", os aspectos relacionados ao processo do trabalho de parto foram os mais questionados. E sobre o "Período Pós-parto" as dúvidas foram sobre aleitamento materno e contracepção (Tabela III). 
Tabela III - Descritores das teleconsultorias realizadas pelo NUTES/UFPE. Recife/PE 2017.

\begin{tabular}{lll}
\hline Descritores & $(\mathbf{n = 9 0 6 )}$ & $\%$ \\
\hline Gravidez & $\mathbf{7 0 1}$ & $\mathbf{7 7 , 3 7}$ \\
Cuidado Pré-natal & 54 & 5,96 \\
Nutrição na gestação & 49 & 5,41 \\
Hipertensão gestacional & 45 & 4,97 \\
Infecção do trato urinário & 28 & 3,09 \\
Administração de medicamentos & 23 & 2,54 \\
Diabetes gestacional & 21 & 2,32 \\
Gravidez de Alto Risco & 20 & 2,21 \\
Neoplasias da Mama; Mastectomia & 19 & 2,10 \\
Sífilis Congênita & 16 & 1,77 \\
Sulfato Ferroso e ácido fólico & 15 & 1,66 \\
Ameaça de Aborto & 15 & 1,66 \\
Exercício físico na gestação & 15 & 1,66 \\
Outros & 381 & 42,05 \\
\hline Gravidez na adolescência & $\mathbf{1 2}$ & $\mathbf{1 , 3 2}$ \\
Complicações na Gravidez & 5 & 0,55 \\
Outros & 7 & 0,77 \\
\hline Parto & $\mathbf{4 0}$ & $\mathbf{4 , 4 2}$ \\
Trabalho de Parto & 18 & 1,99 \\
Placenta & 7 & 0,77 \\
Parto prematuro & 3 & 0,33 \\
Aminiorrexe & 2 & 0,22 \\
Outros & 10 & 1,11 \\
\hline Período pós-parto & $\mathbf{1 5 2}$ & $\mathbf{1 6 , 7 8}$ \\
Aleitamento materno & 79 & 8,72 \\
Contracepção & 20 & 2,21 \\
Outros & 53 & 5,85 \\
\hline Não informado & $\mathbf{1}$ & $\mathbf{0 , 1 1}$ \\
\hline Total Geral & $\mathbf{9 0 6}$ & $\mathbf{1 0 0 , 0 0}$ \\
\hline & & \\
\hline & 53 & \\
\hline
\end{tabular}

Discussão

A teleassistência vem se mostrando como um instrumento de suporte para segunda opinião entre profissionais de saúde, em especial, aos da atenção primária, onde o acesso a especialidades e a serviços de maior complexidade (níveis secundário e terciário) é escasso $[13,14]$. Um aspecto importante a ser ressaltado é o caráter multi e interdisciplinar das teleconsultorias que em geral, busca envolver mais de uma especialidade nas respostas enviadas por entender a importância da integralidade do cuidado com vistas a restabelecer a condição de saúde do indivíduo.

Em se tratando da atenção obstétrica entende-se que a gestação representa um marco na vida de um grupo, família e em especial de uma mulher. Nenhum outro evento na vida de um ser humano é tão complexo, pois este repercute profundamente nos planos físico, mental, emocional e social, além de representar uma mudança definitiva de papéis [15]. Assim, a atenção multidisciplinar proporcionada pela teleassistência nesta área reforça a compreensão da complexidade deste evento e da importância do cuidado integral em saúde e oportuniza melhores condições de assistência à gestante, família e feto, seja durante a gravidez, parto e puerpério [1].

Os enfermeiros e os agentes comunitários de saúde foram os profissionais que mais enviaram solicitações por serem estes o maior público que utiliza a telessaúde. Fato semelhante foi encontrado em diversos estudos que encontraram que estas categorias de profissionais buscam utilizar com maior frequência a ferramenta da telessaúde por serem eles os principais intermediadores entre a população assistida e o acesso aos cuidados de saúde [13,16-19].

Este público trouxe as questões de sua população assistida para serem discutidas com os teleconsultores, o que proporciona resolutividade de maneira ágil para os casos nos quais são necessários intervenções e/ou encaminhamentos para especialistas e reforço à educação em saúde quando esclarecidas dúvidas destes profissionais acerca de quadros clínicos ou de seu processo de trabalho. A eficiência do retorno das teleconsultorias ficou evidente tanto pelo 
tempo de retorno aos solicitantes (menor que um dia) quanto pela resolução dos casos já na primeira teleconsulta. Isto representa um ganho para a população assistida no que diz respeito ao tempo de acesso aos especialistas, implementação do tratamento adequado em tempo oportuno quando comparados aos atendimentos presenciais convencionais que, por vezes, demandam um tempo mínimo de um mês para atendimento [16,20].

Salienta-se que apesar do planejamento do solicitante em encaminhar a paciente para serviços de mais alta complexidade, apenas $7,6 \%$ se fez realmente necessário. Além disso, o grau de satisfação dos profissionais que retornaram com um feedback sobre as respostas dos teleconsultores se mostrou alto, sendo classificado como Ótimo e Bom. Contudo, registra-se ainda o baixo retorno sobre os desfechos dos casos e/ou resolução das questões por parte dos solicitantes. Este retorno ao teleconsultor e ao serviço é de extrema importância para aprimoramento e controle de qualidade dos serviços. Observa-se através de outras experiências no Brasil, que há esse baixo retorno dos solicitantes, dificultando a avaliação dos serviços de telessaúde [16,20-24].

As teleconsultorias permitem caracterizar 0 profissional solicitante e, consequentemente, qualificam os atendimentos ao usuário, evitando também os encaminhamentos desnecessários ou qualificando aqueles encaminhamentos que se fazem necessários. Isto tem impacto importante no que tange aos aspectos relacionados à segurança e deslocamento dos pacientes ao deslocar-se do interior dos municípios longínquos até os centros urbanos, além da exposição às longas filas de espera e superlotação dos hospitais terciários para atendimentos que a atenção básica resolveria com a ajuda da teleassistência através de seus especialistas $[7,14,16]$.

O uso desta tecnologia tem apresentado impactos na qualificação da assistência prénatal, uma vez que a segunda opinião auxilia os profissionais não-especialistas na condução e resolução das principais intercorrências que por ventura ocorram durante o período gravídicopuerperal. Países desenvolvidos e em desenvolvimento já tem comprovado em suas experiências o impacto da telessaúde na redução da morbimortalidade através da detecção precoce de eventos adversos [18-21].

Adicionalmente, a telessaúde impõe contribuições relevantes em se tratando das estratégias de ensino-aprendizagem em saúde através do uso de recursos tecnológicos de informação, como a webconferência, teleconsultorias, entre outros, para colaborar com a formação permanente dos profissionais. Todas as categorias profissionais podem se beneficiar dessas contribuições da teleassistência. Ademais, observa-se que a categoria mais participativa (tanto na solicitação quanto na resposta da teleconsultoria) é a Enfermagem $[11,17,18]$.

O tema das questões enviadas aos teleconsultores eram especialmente relacionadas à condutas assistenciais que devem ser tomadas durante o pré-natal, tais como prescrição de medicação e protocolos relativos à conduta profissional. Também foi enviado número expressivo de casos relacionados à prevenção e/ou detecção e/ou tratamento relacionados à hipertensão gestacional. Em se tratando do período pós-parto, questões relacionadas à amamentação foram as mais presentes.

Dessa forma, percebe-se que o serviço de teleconsultoria, por contribuir com a qualificação da assistência prestada às mulheres em seus diversos ciclos de vida, e em especial no período gestacional, possui um potencial para favorecer a redução da taxa de mortalidade materna $[18,19]$.

Apesar de todas as contribuições aqui registradas, observa-se como limitação para o uso desta tecnologia a escassez e/ou entrave relacionado ao acesso à internet em alguns locais distantes dos grandes centros urbanos. Outra limitação que ultrapassa o poder de atuação da telessaúde está na resolução local dos casos. A exemplo disto, salienta-se a dificuldade encontrada pelos profissionais da atenção básica em seguir as intervenções e condutas propostas quanto a prescrição de um tratamento e/ou a solicitação de um exame, pois não dispõe destes recursos ou há demora na entrega dos resultados.

Conclusão

A telessaúde é uma importante ferramenta de suporte ao enfermeiro, particularmente para aqueles que estão geograficamente distantes de centros de saúde. $O$ estudo demonstrou que o uso da teleassistência tem comprovado potencial para qualificação permanentemente da equipe da atenção básica, em especial ao enfermeiro, melhorando a qualidade e resolubilidade 
do atendimento às mulheres no ciclo gravídico puerperal, otimizando assim a utilização dos recursos do sistema público de saúde.

Dessa forma, a teleassistência vem cumprindo o seu papel de apoiar os profissionais que necessitam de uma segunda opinião dos especialistas para conduzir as situações clínicas e de processo de trabalho vivenciadas no seu cotidiano de trabalho. Isto tem sido observado pela eficiência e satisfação observada dos serviços aqui analisados.

A teleassistência também se mostra uma área promissora de atuação para os enfermeiros obstetras. Através das teleconsultorias, a enfermagem obstétrica pode apoiar os profissionais não especialistas a melhor atender às mulheres em todo o ciclo gravídico puerperal, desde a preconcepção até o término do puerpério, estendendo-se também à atenção aos bebês e familiares.

A contribuição dos resultados deste estudo auxilia para o direcionamento de ações de aprimoramento profissional voltadas às demandas assistenciais vivenciadas pelos enfermeiros e outros profissionais na Atenção Básica relacionadas ao período gravídico puerperal, visando avanços na assistência integral à mulher e melhores índices de saúde.

A enfermagem obstétrica pode se beneficiar bastante dos serviços de teleassistência conforme pode ser observado pelos números da experiência do NUTES/UFPE. A disponibilidade de acesso a especialistas e a qualidade das teleconsultorias se mostra promissora para redução das dúvidas relativas ao período gravídico-puerperal, impactando indiretamente, na redução de complicações obstétricas evitáveis. Como trabalhos futuros, será analisado o impacto do uso da telessaúde na redução dos impactos da morbimortalidade materna e neonatal.

Agradecimentos

Agradecemos ao Ministério da Saúde e à Telessaúde Brasil Redes pelo financiamento do Programa e ao Núcleo de Telessaúde da Pernambuco (NUTES/HC/UFPE) pela sua anuência ao estudo.

1. Brasil M da S. Protocolos da Atenção Básica: Saúde das Mulheres [Internet]. 1a. Ministério da Saúde B, editor. Brasília: Ministério da Saúde. Secretaria de Atenção a Saúde. Departamento de Atenção Básica; 2016. 230 p.

2. Brasil M da S. Painel de Monitoramento da Mortalidade Materna [Internet]. 2017 [cited 2017 Jan 4]. Available from: http://svs.aids.gov.br/dashboard/mortalidade/materna.show.mtw

3. WHO WHO. Recomendações da OMS sobre cuidados pré-natais para uma experiência positiva na gravidez. 1st ed. WHO WHO, editor. Genebra: World Health Organization; 2016. 10 p.

4. Ministério da Saúde B. Telessaude Brasil Redes [Internet]. Brasil; 2013. [citado 2017 Abr 12]. Disponível em: http://www.telessaudebrasil.org.br/

5. Wen CL. Telemedicina e Telessaúde - um panorama no Brasil. Informática Pública 2008;2(10):7-15.

6. Cruz ELD, Novaes MA, Machiavelli JL, Menezes VA de. Caracterização dos seminários por webconferência sobre saúde do adolescente e jovem da rede de núcleos de telessaúde de Pernambuco. Rev Bras Saúde Materno Infantil 2012;12(1):83-90.

7. Alves DS, Sanches LMP, Novaes MA, Lopes MHBM. Estratégias de telessaúde no suporte ao enfermeiro do PSF no estado de Pernambuco. In: 120 Congresso Brasileiro dos Conselhos de Enfermagem, CBCENF. Belo Horizonte; 2009. p. 12.

8. Oliviera DG, Frias PG, Vanderlei LCM, Vidal SA, Novaes MA, Souza WV. Análise da implantação do Programa Telessaúde Brasil em Pernambuco, Brasil: estudo de casos. Cad Saúde Pública 2015;31(11):2379-89.

9. Polinski JM, Barker T, Gagliano N, Sussman A, Brennan TA, Shrank WH. Patients' satisfaction with and preference for telehealth visits. Journal of General Internal Medicine 2016;31(3):269-75.

10. Medronho. Epidemiologia. 2nd ed. Rio de Janeiro: Atheneu; 2009.

11. NUTES/UFPE. HealthNET 2.0 [Internet]. [citado 2017 Aug 26]. Disponível em: http://www.nutes.ufpe.br/healthnet/index.html\# 
12. Silva TPS, Barros MBSC, Silva KCL, Alves DS, Batista MC de M, Diniz PRB et al. Plataforma de telessaúde - healthnet: uma ferramenta para teleconsultorias assíncronas. J Bras Tele 2013;2:123.

13. Ministério da Saúde. Portaria No 2.546, de 27 de outubro de 2011 [Internet]. 2011 [citado 2017 Nov 16]. Disponível em: http://bvsms.saude.gov.br/bvs/saudelegis/gm/2011/prt2546_27_10_2011.html

14. Alves DS, Sanches LMP, Novaes MA, Lopes MHBM. Fale com o enfermeiro: serviço de segunda opinião à distância para atenção primária. In: 120 Congresso Brasileiro dos Conselhos de Enfermagem, CBCENF. Belo Horizonte; 2009.

15. Joshi A, Novaes MA, lyengar S, Machiavelli JL, Zhang J, Vogler $R$ et al. Evaluation of a tele-education programme in Brazil. Journal of Telemedicine and Telecare 2011;17(7):341-5.

16. Silva MG, Shimo AKK. Influência da iluminação nas expressões emocionais de parturientes: ensaio clínico randomizado. Acta Paul Enferm 2017;30(3):217-26.

17. Diniz PRB, Ribeiro Sales FJ, de Araújo Novaes M. Providing telehealth services to a public primary care network: the experience of RedeNUTES in Pernambuco, Brazil. Telemedicine journal and e-health: the official journal of the American Telemedicine Association [Internet]. 2016 Aug [citado 2016 Sep 24];22(8):694-8. Disponível em: http://www.ncbi.nlm.nih.gov/pubmed/26953495

18. Santos MVR, Oliveira DC, Novaes MA. A telehealth strategy for increasing adherence in the treatment of hypertension in primary care. Telemedicine journal and e-health: the official journal of the American Telemedicine Association [Internet]. 2013[citado 2014 Feb 9];19(4):241-77. Disponível em: http://online.liebertpub.com/doi/abs/10.1089/tmj.2012.0036

19. Pai N, Supe P, Kore S, Nandanwar YS, Hegde A, Cutrell E, et al. Using automated voice calls to improve adherence to iron supplements during pregnancy. Proceedings of the Sixth International Conference on Information and Communication Technologies and Development Full Papers - ICTD '13 - volume 1 [Internet]. 2013;153-63. [citado 2017 abr 12]. Available from: http://dl.acm.org/citation.cfm?doid=2516604.2516608

20. Alves DS, Novaes MA. Profile of the nursing team and level of satisfaction in relation to the usability of the Electronic Patient's Record. Journal of Nursing UFPE 2013;7(1):14352.

21. Sanches LMP, Alves DS, Lopes MHBM, Novaes MA. The practice of telehealth by nurses: an experience in primary healthcare in Brazil. Telemedicine journal and e-health: the official journal of the American Telemedicine Association [Internet]. 2012 Nov 7 [cited 2014 Feb 20];18(9):679-83. Available from: http://online.liebertpub.com/doi/abs/10.1089/tmj.2012.0011

22. Prado C, Silva IA, Soares AVN, Aragaki IMM, Shimoda GT, Zaniboni VF et al. Teleamamentação no Programa Nacional de Telessaúde no Brasil: a experiência da Telenfermagem. Rev Esc Enferm USP 2013;47(4):990-6.

23. Correia AD da MS, Dobashi BF, Gonçalves CCM, Monreal VRFD, Nunes EA, Haddad $\mathrm{PO}$ et al. Teleodontologia no programa nacional telessaúde Brasil redes: relato da experiência em Mato Grosso Do Sul. Revista da ABENO 2014;14(1):17-29.

24. Correia ADMS, Dobashi BF, Gonçalves CCM, Monreal VRFD, Borsoi BM, Kanomata MN et al. Telenfermagem e teleodontologia: relato da experiência do telessaúde Brasil redes em Mato Grosso do Sul. 2012. [cited 2018 May 4]; Available from: http://www.convibra.com.br/upload/paper/2013/63/2013_63_6379.pdf 